\title{
Omnidirectional absorption in nanostructured metal surfaces
}

William T.V. Teperik, ${ }^{1}$ F.J.Garcia de Abajo, ${ }^{1,2}$ A. G. Borisov, ${ }^{3}$ Y. Sugawara, ${ }^{4}$ J. J.

Baumberg, ${ }^{4}$ M. Abdelsalam ${ }^{5}$ \& P. N. Bartlett ${ }^{5}$

${ }^{1}$ Donostia International Physics Center, Aptdo. 1072, 20080 San Sebastian, Spain

${ }^{2}$ Instituto de O' ptica - CSIC, Serrano 121, 28006 Madrid, Spain

${ }^{3}$ Laboratoire des Collisions Atomiques et Mol'eculaires, UMR 8625 CNRS-

Universit'e Paris-Sud, 91405 Orsay Cedex, France

${ }^{4}$ Department of Physics and Astronomy, University of Southampton, Southampton, SO17 1BJ,United Kingdom

${ }^{5}$ School of Chemistry, University of Southampton, Southampton UK SO17 1BJ. Email:pnb@soton.ac.uk

\section{Please cite this paper as:}

Nature Photonics, 2008, 299-301

The publisher's version of this paper is available here:

http://dx.doi.org/10.1038/nphoton.2008.76

\section{Related articles by Prof Phil Bartlett can be found below:}

P. N. Bartlett, J. J. Baumberg, P. R. Birkin, M. A. Ghanem and M. C. Netti, “Highly Ordered Macroporous Gold and Platinum Films formed by Electrochemical Deposition through Templates Assembled from Submicron Diameter Monodisperse Polystyrene Spheres" Chem. Mater., 14, 2199-2208 (doi: 10.1021/cm011272j)

T. A. Kelf, Y. Sugawara, R. M. Cole, J. J. Baumberg, M. E. Abdelsalam, S. Cintra, S. Mahajan, A. E. Russell, and P. N. Bartlett, "Localized and delocalized plasmons in metallic nanovoids", Phys. Rev. B, 74, Art. No. 245415, 1-12 (doi:10.1103/PhysRevB.74.245415)

R. M. Cole, J. J. Baumberg, F.J. Garcia de Abajo, S. Mahajan, M. Abdelsalam and P. N. Bartlett, "Understanding Plasmons in Nanoscale Voids", Nano Lett., 7, 2007, 2094-2100. (doi: 10.1021/nl0710506)

N. N. Lal, B. F. Soares, J. K. Sinha, F. Huang, S. Mahajan, P. N. Bartlett, N. C. Greenham, and J. J. Baumberg, "Enhancing solar cells with localized plasmons in nanovoids", Optics Express, 19, 2011, 11256-11263. (doi: 10.1364/OE.19.011256)

F. M.Huang, J. K. Sinha, N. Gibbons, P. N. Bartlett and J. J. Baumberg, "Direct assembly of three-dimensional mesh plasmonic rolls", Appl. Phys. Lett., 100, 2012, 193107 (1-4). (doi:10.1063/1.4711923)

N. N. Lal, H. Zhou, M. Hawkeye, J. K. Sinha, P. N. Bartlett, G. A. J. Amaratunga and J. J. Baumberg, "Using spacer layers to control metal and semiconductor absorption in ultrathin solar cells with plasmonic substrates", Phys. Rev. B, 85, 2012, 245318(1-10). (doi: 10.1103/PhysRevB.85.245318) 


\section{Omnidirectional absorption in nanostructured metal sur- faces}

T. V. Teperik, ${ }^{1}$ F. J. García de Abajo, ${ }^{1,2}$ A. G. Borisov, ${ }^{3}$ Y. Sugawara, ${ }^{4}$ J. J. Baumberg, ${ }^{4}$ M. Abdelsalam $^{5} \&$ P. N. Bartlett ${ }^{5}$

${ }^{1}$ Donostia International Physics Center, Aptdo. 1072, 20080 San Sebastian, Spain

${ }^{2}$ Instituto de Óptica - CSIC, Serrano 121, 28006 Madrid, Spain

${ }^{3}$ Laboratoire des Collisions Atomiques et Moléculaires, UMR 8625 CNRS-Université Paris-Sud, 91405 Orsay Cedex, France

${ }^{4}$ Department of Physics and Astronomy, University of Southampton, Southampton, SO17 1BJ, United Kingdom

${ }^{5}$ Department of Chemistry, University of Southampton, Southampton, SO17 1BJ, United Kingdom

Currently-available light absorbers give far from the optimal black-body performance. The need for new efficient absorbers is particularly acute on the microscale, where they can play a significant role in preventing cross-talk between optical interconnects, and also as thermal light-emitting sources. Several efforts have been made in this context to achieve near-total but directionally-dependent absorption using periodic grating structures ${ }^{1-7}$. However, the ability to absorb light completely for any incident direction of light remains a challenge. Here we show that total omnidirectional absorption of light can be achieved in nanostructured metal surfaces sustaining localized optical excitations. The effect is realized over a full range of 
incident angles and can be tuned throughout visible and near-infrared regimes by scaling the nanostructure dimensions. We suggest that surfaces displaying omnidirectional absorption will play a key role in devising efficient photovoltaic cells, in which the absorbed light goes into electron-hole pair production. The ability to shield spurious light through total absorption is also important in the design of highly-integrated optical devices and for efficient thermal light micro-emitters thanks to Kirchhoffs law ${ }^{8}$.

Planar metals make excellent mirrors, but they become poor reflectors when structured at subwavelength scales, like in the case of black silver ${ }^{9-11}$ and random particle arrays ${ }^{12}$. This behavior can be largely attributed to absorption triggered by plasmon excitations, although corrugation is capable by itself to suppress reflection even in dielectrics, like in the 'moth eye' phenomenon occurring at the interface between air and the cornea ${ }^{13}$. Actually, a continued effort has been carried out over the last decades to show that surface excitations at optical ${ }^{1,2,6,14}$, infrared ${ }^{5,15}$, and microwave frequencies ${ }^{7}$ can produce full absorption using linear gratings. These works have been extended theoretically $y^{4,16,17}$ and experimentally ${ }^{3}$ to doubly-periodic gratings as well. However, absorption in gratings relies on delocalized surface excitations that are highly sensitive to the angle of incidence. This directionality prevents application to photovoltaic cells and microscale lighting where wide collection and emission angles are generally required.

Here, we provide experimental and theoretical support for an omnidirectional absorption effect relying on the excitation of localized plasmons. In an intuitive picture, we can argue that optical resonances trap light energy for a period of time proportional to their quality factor, so that 
the trapped light is absorbed due to dissipation in metal when the dwell time is sufficiently large. We specifically focus on localized plasmon excitations because they can be efficiently excited over wide ranges of incidence angles, yielding the total absorption effect not only omnidirectional but also polarization independent. We demonstrate the feasibility of this concept using nanoporous metal surfaces prepared by electrochemical deposition of gold over a compact monolayer of latex spheres supported on a gold substrate. The latex is later removed to make empty spherical voids buried in gold (Fig. 1A). This sample fabrication procedure has been extensively explained elsewhere $^{18}$. These localized plasmons are coupled to the external light, so that radiative decay contributes to their finite lifetime and they must be understood as resonances in that sense. However, it is customary to denote them plasmons ${ }^{19}$ because like surface-plasmon polaritons they involve mixing of light and collective valence electron excitations in the metal.

An optically thick sample exhibiting a surface resonance can be made to absorb light completely if it is designed in such a way that the radiative decay rate of the resonance equals the rate associated to dissipation (rate equipartition condition), provided diffraction and polarization conversion are suppressed. This stimulating result finds its explanation in the general principles of scattering theory developed in quantum mechanics, as shown in the Supporting Online Material (SOM). Under these conditions, the reflectivity reduces to

$$
R=\frac{\left(\omega-\omega_{0}\right)^{2}}{\left(\omega-\omega_{0}\right)^{2}+\Gamma^{2} / 4}
$$

where $\Gamma$ is the resonance width. As anticipated, the reflectivity vanishes at the resonance frequency $\omega_{0}$. 
We first investigate the optical spectra of nanoporous metal surfaces under normal incidence. For fixed diameter of the nanovoids $(D=500 \mathrm{~nm})$, this system has a geometrical degree of freedom that can be adjusted to fulfill the rate equipartition condition: the nominal thickness $t$ of metal covering the latex spheres in the fabrication of these samples (see Fig. 1A), which is varied around values close to one diameter of the spheres $(t \sim D)$. As the metal grows around the top of the spheres, the aperture on the top does not completely close but smoothly decreases in a cone, allowing fine tuning of the optical coupling to the outside. The corresponding measured absorption spectra (Fig. 1B) show a prominent feature to the red of the fully-buried-void dipolar plasmon (see arrow). This shift is caused by leakage of the void plamons into the light continuum of the halfempty space above the surface ${ }^{20}$, so that the magnitude of the shift decreases as $t$ increases (i.e., as the void is buried deeper in metal). This decrease in redshift must be accompanied by smaller radiative decay rate, so that at an specific value $(t=1.08 D)$ the rate equipartition condition is fulfilled and the maximum absorption becomes close to $100 \%$.

With a hexagonal lattice of period $500 \mathrm{~nm}$, the wavelength of the onset for diffraction (433 $\mathrm{nm})$ is well below the minimum wavelength explored in Fig. $1(689 \mathrm{~nm})$. Furthermore, polarization conversion during specular reflection is forbidden along the 12 high-symmetry azimuthal directions of the hexagonal lattice and so is the contribution arising from excitation of localized dipole modes of the voids. Therefore, the incident light is either absorbed or specularly reflected with preservation of polarization, so that the rate equipartition condition is sufficient to guarantee total absorption, as shown in the SOM. 
We have simulated absorption spectra by rigorous solution of Maxwell's equations using a layer-Korringa-Kohn-Rostoker (KKR) approach for fully-buried voids ${ }^{17,21,22}$. However, our samples have openings at the top of the voids (see Fig. 1A) and possibly in the touching region between adjacent voids as well. The effect of partial penetration of the electromagnetic field through these openings has been phenomenologically accounted for by describing the metal using a MaxwellGarnett dielectric function ${ }^{23}$ formed by $55 \%$ empty pores infiltrated in gold, with the latter represented by measured optical data ${ }^{24}$. The calculated results (Fig. 1C) show good qualitative agreement with the measurements, although the redshift and width of the resonant absorption features are larger in the experiment, presumably as a result of stronger interaction of the void plasmon with SPPs and the half-empty space above the sample through the openings. Nonetheless, the same evolution of the maximum absorption with $t$ is observed in experiment and theory, with an optimum metal thickness at which absorption reaches $100 \%$.

The nanoporous metal surfaces of Fig. 1A can simultaneously support delocalized surfaceplasmon polaritons (SPPs) and localized void plasmons. The former cannot couple directly to incident light ${ }^{25}$, although the array of voids provides an efficient interaction mechanism ${ }^{26,27}$. However, it is the localized void plasmons that will provide the omnidirectionality that we are pursuing.

The effect of nearly-total absorption observed in Fig. 1 persists for a relatively large range of incidence angles up to $\sim 40^{\circ}$, above which the effect of SPPs in the flat metal interface becomes apparent and produces non-vanishing absorption (se Fig. S1 in the SOM). This problem can be solved by infiltrating the voids with a dielectric medium, so that the void plasmon is brought down 
in energy to a region where it does not interact with the delocalized SSPs, as illustrated in Fig. 2 for gold containing a compact layer of silica inclusions $(\epsilon=2.1)$.

Without the influence of SPPs, the total absorption effect extends much further away from normal incidence (Fig. 2A) for arbitrary azimuthal directions (Fig. 2B). This behavior is observed both for TM polarization (Fig. 2) and for TE polarization (not shown). The latter is particularly clear after total absorption is achieved at normal incidence, because the electric field continues to be directed parallel to the surface for any direction of incidence. These results demonstrate that the effect is nearly omnidirectional and independent of polarization.

An additional advantage of staying away from SPPs is that light diffraction is prevented, because it involves non-zero-order scattering into external light modes assisted by momentum transfer to the lattice, but the SPPs lie very close to the light dispersion relation of gold at nearinfrared frequencies.

Photovoltaic cells constitute a natural field of application of total omnidirectional light absorption (with absorbing semi-conductors, rather than metals) because they require broad collection angles. In this direction, the localized plasmons of randomly-arranged, polymorphic metallic nanoparticles have recently been found to enhance the observed photocurrent ${ }^{19}$, and we expect that this effect is further improved by relying instead on regular structures exhibiting plasmons of well defined wavelength, as those used here for omnidirectional total absorption. However, the effect takes place within a relatively narrow spectral range, although this could be widened by combining layered structures of different periodicity. Light shielding of micro-photonic devices can also 
benefit from the reported effect, for instance to prevent cross-talk between optical interconnects. Finally, as a consequence of Kirchhoff's law ${ }^{8}$, our nanostructured metal surfaces should exhibit omnidirectional black-body emission at a resonant frequency that can be tuned by varying the size of the dielectric inclusions, thus resulting in spectrally-narrow wide-angle thermal emitters.

February 7, 2008

1. Maystre, D. \& Petit, R. Brewster incidence for metallic gratings. Opt. Commun. 17, 196-200 (1976).

2. Hutley, M. C. \& Maystre, D. The total absorption of light by a diffraction grating. Opt. Commun. 19, 431-436 (1976).

3. Nevière, M., Maystre, D., MacPhedran, R., Derrick, G. \& Hutley, M. On the total absorption of unpolarized monochromatic light. In Proc. of ICO-11 Conference, Madrid, Spain, 609-612 (1978).

4. Derrick, G. H., McPhedran, R. C., Maystre, D. \& Nevière, M. Crossed gratings: A theory and its applications. Appl. Phys. 18, 39-52 (1979).

5. Greffet, J. J. et al. Coherent emission of light by thermal sources. Nature 416, 61-64 (2002).

6. Collin, S., Pardo, F., Teissier, R. \& Pelouard, J. L. Efficient light absorption in metalsemiconductormetal nanostructures. Appl. Phys. Lett. 85, 194-196 (2004). 
7. Y. P. Bliokh, J. F. \& Slutsker, Y. Z. Total absorption of an electromagnetic wave by an overdense plasma. Phys. Rev. Lett. 95, 165003 (2005).

8. Reif, F. Fundamentals of Statistical and Thermal Physics (McGraw-Hill, New York, 1965).

9. Kohlschutter, V. \& Toropoff, T. Information on the forms of electrolytically precipitated metals. i black silver. Z. Elektrochem. Angew. Chem. 19, 161-168 (1913).

10. Hunderi, O. \& Myers, H. P. Optical absorption in partially-disordered silver films. J. Phys. F 3, 683-690 (1973).

11. Wang, C. M., Chen, Y. C., Lee, M. S. \& Chen, K. J. Microstructure and absorption property of silver-black coatings. Jpn. J. Appl. Phys. Part 1 39, 551-554 (2000).

12. S. Kachan, O. S. \& Ponyavina, A. High-absorbing gradient multilayer coatings with silver nanoparticles. Phys. Rev. B 84, 281-287 (2006).

13. Clapham, P. B. \& Hutley, M. C. Reduction of lens reflexion by the "moth eye" principle. Nature 24, 281-282 (1973).

14. Popov, E. \& Tsonev, L. Comment on 'resonant electric field enhancement in the vicinity of a bare metallic grating exposed to s-polarized light by a. a. maradudin and a. wirgin'. Surf. Sci. Lett. 271, L378-L382 (1992).

15. Popov, E., Tsonev, L. \& Maystre, D. Lamellar metallic grating anomalies. Appl. Opt. 33, 5214-5219 (1994). 
16. Tan, W. C., Sambles, J. R. \& Preist, T. W. Double-period zero-order metal gratings as effective selective absorbers. Phys. Rev. B 61, 13177-13182 (1999).

17. Teperik, T. V., Popov, V. V. \& García de Abajo, F. J. Void plasmons and total absorption of light in nanoporous metallic films. Phys. Rev. B 71, 085408 (2005).

18. Abdelsalam, M., Bartlett, P. N., Baumberg, J. J. \& Coyle, S. Preparation of arrays of isolated spherical cavities by self-assembly of polystyrene spheres on self-assembled pre-patterned macroporous films. Adv. Mater. 16, 90 (2004).

19. Pillai, S., Catchpole, K. R., Trupke, T. \& Green, M. A. Surface plasmon enhanced silicon solar cells. J. Appl. Phys. 101, 093105 (2007).

20. Cole, R. M. et al. Understanding plasmons in nanoscale voids. Nano Lett. 7, 2094-2100 (2007).

21. Stefanou, N., Yannopapas, V. \& Modinos, A. Heterostructures of photonic crystals: Frequency bands and transmission coefficients. Comput. Phys. Commun. 113, 49-77 (1998).

22. Stefanou, N., Yannopapas, V. \& Modinos, A. Multem 2: A new version of the program for transmission and band-structure calculations of photonic crystals. Comput. Phys. Commun. 132, 189-196 (2000).

23. Jackson, J. D. Classical Electrodynamics (Wiley, New York, 1999).

24. Johnson, P. B. \& Christy, R. W. Optical constants of the noble metals. Phys. Rev. B 6, 43704379 (1972). 
25. Raether, H. Surface Plasmons on Smooth and Rough Surfaces and on Gratings, vol. 111 of Springer Tracks in Modern Physics (Springer-Verlag, Berlin, 1988).

26. Teperik, T. V. et al. Strong coupling of light to flat metals via a buried nanovoid lattice: The interplay of localized and free plasmons. Opt. Express 14, 1965-1972 (2006).

27. Kelf, T. A. et al. Localized and delocalized plasmons in metallic nanovoids. Phys. Rev. B 74, 245415 (2006).

Supplementary Information is given in a separate file.

Acknowledgements The authors want to thank Vyacheslav Popov for valuable suggestions and fruitful discussions. This work was supported by the Spanish MEC, under contract NAN2004-08843-C05-05, the EU-FP6, under contract NMP4-2006-016881 "SPANS", and the Russian Academy of Sciences, Russian Foundation for Basic Research (Grant 07-02-91011).

Author Information The authors declare no competing financial interests. Correspondence and requests for materials should be addressed to T.V.T. (swxtetet@ sw.ehu.es) and F.J.G.A. (jga@io.cfmac.csic.es). 
Figure 1 Total light absorption in nanostructured metal surfaces. (A) Sketch and scanning electron microscope (SEM) image of mesoporous gold surfaces used in this work, consisting of a layer of close-packed voids of diameter $D=500 \mathrm{~nm}$ covered with gold up to a thickness $t$. The SEM image is taken at $t<D$ to help visualize the inner part of the nanovoids. (B) Measured absorption spectra of samples with different thicknesses under normal incidence. (C) Calculated spectra under the same conditions as in B. The arrows in $B$ and $C$ mark the energy of the dipole plasmon of a single void in gold.

Figure 2 Omnidirectional light absorption. (Top) Calculated incidence-angle dependence of absorption by a layer of 500-nm closed-packed silica-filled inclusions buried in gold for p-polarized light incident along the $\Gamma-M$ direction of the voids lattice. The metal extends $5 \mathrm{~nm}$ above the top of the inclusions in order to maximize absorption over a wide angular range. (Bottom) Under the same conditions, azimuthal dependence of absorption for $20^{\circ}$ off-normal incidence. The polar-plot insets show the angular behavior of absorption at the frequency of the dipolar void plasmon $\omega=1.27 \mathrm{eV}$. 

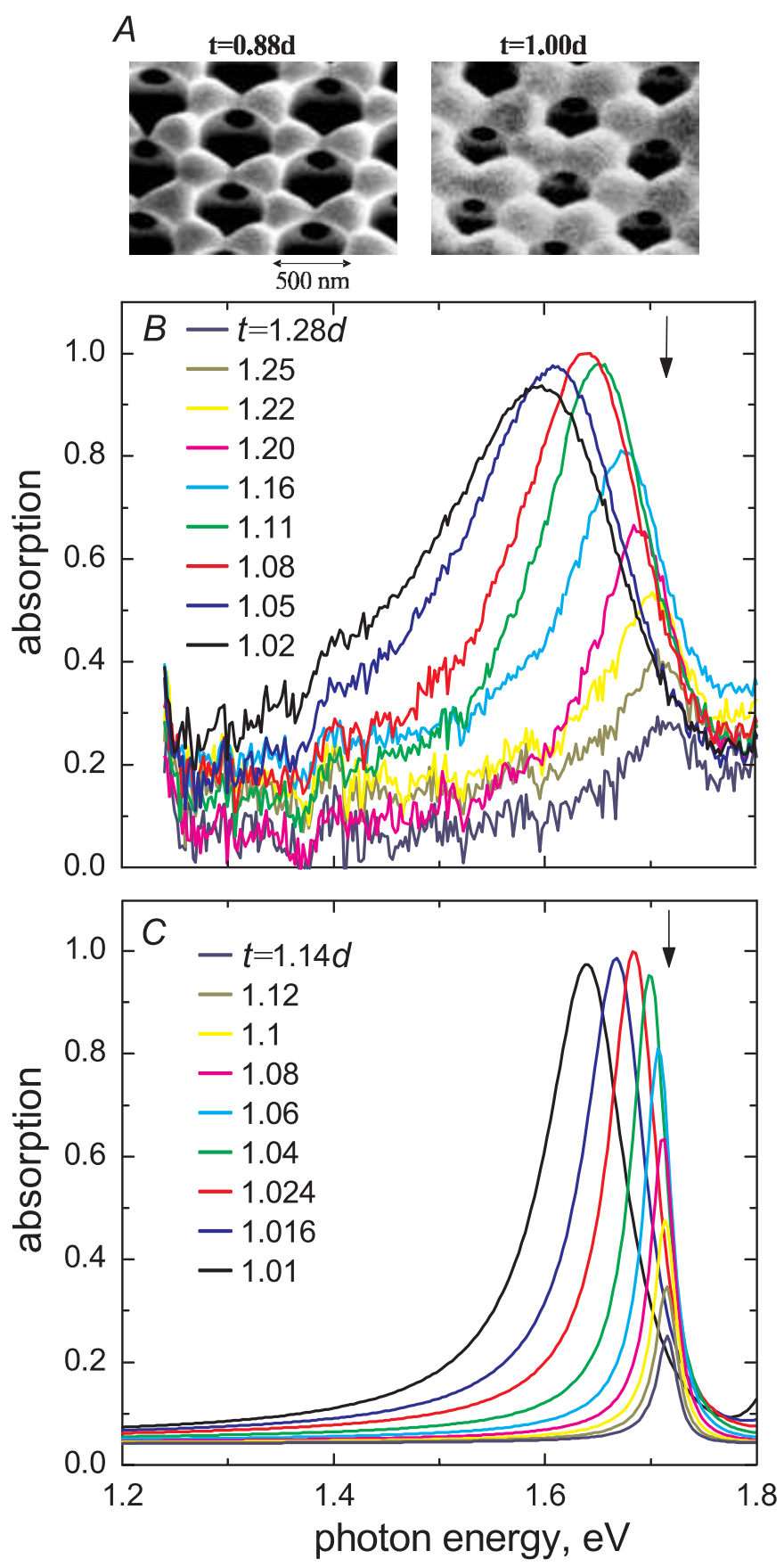

Figure 1: Total light absorption in nanostructured surfaces. (A) Sketch and scanning microscope images of mesoporous gold surfaces used in this work, consisting of a layer of close-packed voids of $500 \mathrm{~nm}$ in diameter covered with metal up to a thickness $t$ normalized to their diameter. (B) Measured absorption spectra of samples with different thicknesses under normal incidence. (C) Calculated spectra under the same conditions as in B. The arrows in B and C mark the energy of the dipole plasmon of a single void in gold. 


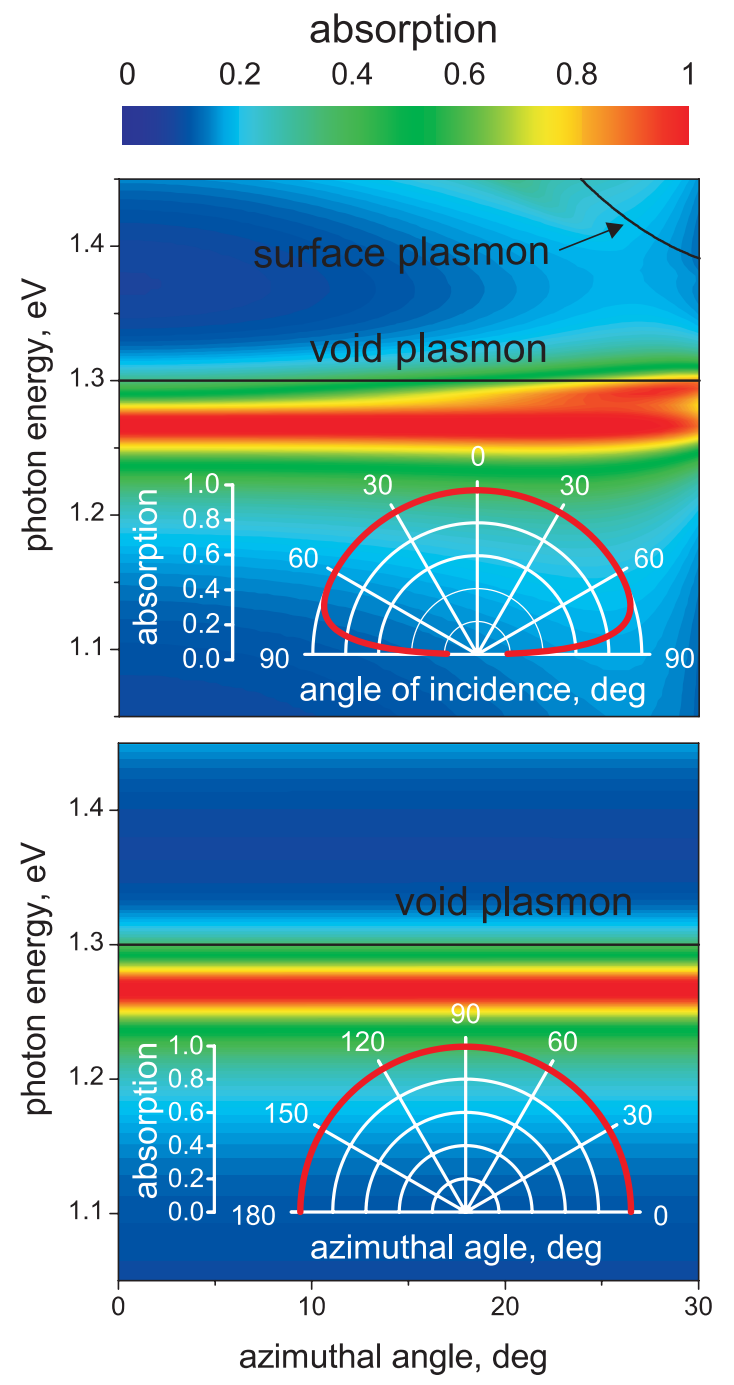

Figure 2: Omnidirectional light absorption. (Top) Calculated incidence-angle dependence of absorption by a layer of 500-nm closed-packed water-filled inclusions buried in gold for ppolarized light directed along the $\Gamma-M$ of the voids lattice. The metal extends $5 \mathrm{~nm}$ above the top of the inclusions in order to maximize absorption over a wide angular range. (Bottom) Under the same conditions, azimuthal dependence of absorption for $20^{\circ}$ off-normal incidence. The polar-plot insets show the angular behavior of absorption at the frequency of the dipolar void plasmon $\omega=1.27 \mathrm{eV}$. 